\title{
SELF-INDUCED STOCHASTIC RESONANCE FOR BROWNIAN RATCHETS UNDER LOAD*
}

\author{
R. E. LEE DEVILLE ${ }^{\dagger}$ AND ERIC VANDEN-EIJNDEN $\ddagger$
}

\begin{abstract}
We consider a Brownian ratchet model where the particle on the ratchet is coupled to a cargo. We show that in a distinguished limit where the diffusion coefficient of the cargo is small, and the amplitude of thermal fluctuations is small, the system becomes completely coherent: the times at which the particle jumps across the teeth of the ratchet become deterministic. We also show that the dynamics of the ratchet-cargo system do not depend on the fine structure of the Brownian ratchet. These results are relevant in the context of molecular motors transporting a load, which are often modeled as a ratchet-cargo compound. They explain the regularity of the motor gait that has been observed in numerical experiments, as well as justify the coarsening into Markov jump processes which is commonly done in the literature.
\end{abstract}

Key words. stochastic resonance, self-induced stochastic resonance, Brownian ratchets, molecular motors

AMS subject classifications. $\quad 60 \mathrm{H} 10,60 \mathrm{~F} 10,92 \mathrm{C} 10,60 \mathrm{G} 35,34 \mathrm{E} 13$

\section{Introduction and motivation}

Rectified thermal diffusion, or the Brownian ratchet, has been used in many contexts as a model for processes at the molecular level; a few examples include RNA polymerase [23], chromosome transport [19], protein translocation [22], and ATP hydrolysis of kinesin [18] (see [9] for a comprehensive list).

The simplest example of a Brownian ratchet is the "perfect" ratchet. Here a particle is moving on a one-dimensional track on which it diffuses freely except at a set of discrete locations (called the "teeth"). At these teeth, the particle is only allowed to pass in one direction (say, from left to right), inducing an average drift of the particle to the right. Another, slightly more sophisticated, example of a Brownian ratchet is that of a particle diffusing in a potential with a sequence of local minima, such that each local minimum has lower energy than the one to its left. The bias encoded in the potential also induces an average drift to the right in the particle's motion.

While a particle in a Brownian ratchet moves with a nonzero mean velocity, its position also has a significant variance. For instance, in the second model above, if the noise is small, each minimum of the potential becomes metastable, and jumps occur amongst minima following Arrhenius' law, i.e. the times between these jumps are approximately independent and exponentially distributed.

The purpose of this paper is to show that a simple modification of Brownian ratchets makes them much more regular. Specifically, we show that if we couple the particle in the ratchet to a heavy cargo which applies a force to this particle, the compound moves deterministically in a distinguished limit when (i) the diffusion coefficient of the cargo is much smaller than that of the particle in the ratchet, and (ii) the energy due to thermal effects is much less than the energy barrier to move the particle from one ratchet tooth to the next while keeping the cargo fixed. Under these

${ }^{*}$ Received: December 13, 2006; accepted (in revised version): April 5, 2007. Communicated by Shi Jin.

${ }^{\dagger}$ Courant Institute of Mathematical Sciences, New York University, New York, NY 10012, USA (deville@cims.nyu.edu).

$\ddagger$ Courant Institute of Mathematical Sciences, New York University, New York, NY 10012, USA (eve2@cims.nyu.edu). 
conditions, the times between successive jumps of the particle have a non-zero mean, while their variance goes to zero. We also show that the dynamics does not depend on the fine structure of the potential we choose: in fact, we show that any potential can be characterized by a few parameters which completely determine the dynamics.

The phenomenon by which regularity arises is that of self-induced stochastic resonance, studied in $[12,11,3,16,5,4,6]$. It can be roughly summarized as follows: if a system is driven by small noise, the escape times of the system from some region are exponentially distributed, and their transition rate is governed by an activation energy. Let us now assume that the system is placed out of equilibrium and parameters are chosen so that it relaxes slowly. Assume also that the activation energy decreases as the system relaxes. Then, during the relaxation phase, there is a specific time at which the timescale for escape events switches from being longer than the relaxation timescale to being shorter, and thus the system jumps reliably at that point. If the jump brings the system back out of equilibrium, the relaxation stage can start over again, and the scenario repeats itself indefinitely. Below we show how this scenario can arise in Brownian ratchets coupled to a load.

The results presented here are relevant in the context of molecular motors, since Brownian ratchets are often used as a model of a motor protein moving along a filament $[14,9]$ and have been shown to give a good phenomenological description of such motors [1]. It has also been observed that having an elastic tether between motor and cargo allows the motor to efficiently transport a cargo with a much smaller diffusion coefficient $[9,15]$, and moreover this can make the motor gait become much more regular [20]. Our results explain why this regularity occurs. They also go some way towards justifying the coarse-graining which is typically done in this field [10]: many common motor protein models ignore the fine structure of the motor and simply model the coarse-grained dynamics empirically via Markov jump processes. In fact, the authors have shown [7] in the context of Markov jump process models of motor proteins that tethering a motor to a heavy cargo with an elastic spring can induce regularity in the motion of the motor and make it jump deterministically. One consequence of the present work is a justification of this coarse-graining.

We now describe the organization of the remainder of this paper. In Section 2 we formulate the problem and describe the equations. In Section 3 we study a certain distinguished limit in great detail and show that it gives rise to regular dynamics of the system. In Section 4 we comment on several implications of the results here to motor protein dynamics in general. In Appendix A we state a precise formulation of the limit described in Section 3 and sketch the proof.

\section{Problem formulation}

Hereafter we refer to the particle in the ratchet as the motor and to the attached load as the cargo. We denote as $x$ the position of the motor and $y$ the position of the cargo. A schematic diagram of the motor-cargo complex is given in Figure 2.1. The potential energy of the system is given by

$$
\phi(x, y)=U(x)+S(|x-y|),
$$

where $U$ represents the potential energy of the motor interacting with the ratchet, and $S$ represents the energy contained in the spring coupling the motor to the cargo. We assume that $U: \mathbb{R} \rightarrow \mathbb{R}$ and $S: \mathbb{R}^{+} \rightarrow \mathbb{R}^{+}$are $C^{2}$, and that $S$ is convex and satisfies $S(0)=S^{\prime}(0)=0$. We further assume that

$$
U(x+A)=U(x)-U_{*},
$$


where $U_{*}>0$, and that $U$ has exactly one local minimum and one local maximum in any interval of the form $[x, x+A)$. Without loss of generality we will assume that the local minima are located at integral multiples of $A$. An example potential is given in figure 2.2 .

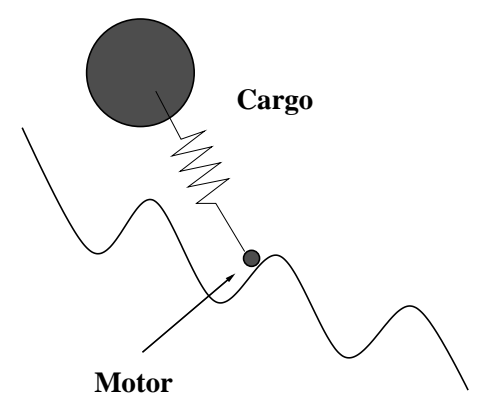

FIG. 2.1. A schematic diagram of the motor-cargo complex. The motor moves in the tilted potential which we describe here, and the cargo is tethered to the motor by a nonlinear spring. We will eventually assume that the diffusion coefficient of the motor is much larger than that of the cargo, which we represent by a "small" motor pulling a "large" cargo.

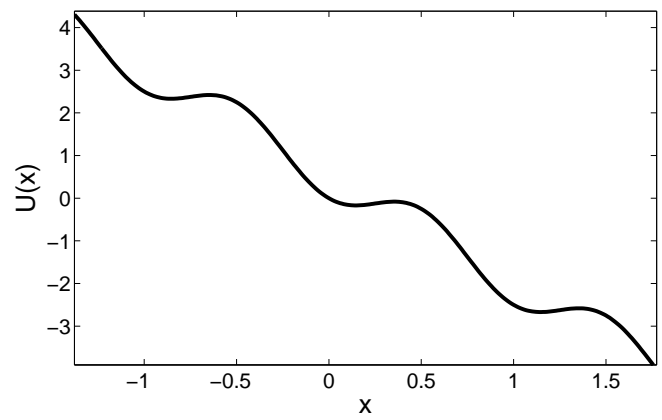

FIG. 2.2. One possible choice of $U(x)$, namely $U(x)=1 / 2(1-\cos (2 \pi x)-5 x)$ (cf. Figure 3.1).

Following [9], we model the dynamics of both the cargo and motor as overdamped due to their microscopic size. In this limit, the dynamics of both the cargo and the motor can be described by the following pair of coupled SDEs:

$$
\begin{aligned}
& d x=-\frac{D_{M}}{k_{B} T} \frac{\partial \phi}{\partial x} d t+\sqrt{2 D_{M}} d W_{t}^{x}, \\
& d y=-\frac{D_{C}}{k_{B} T} \frac{\partial \phi}{\partial y} d t+\sqrt{2 D_{C}} d W_{t}^{y},
\end{aligned}
$$

where $W_{t}^{x}, W_{t}^{y}$ are independent Brownian motions modeling the effect of thermal noise on the compound, and $D_{M}, D_{C}$ are the diffusion coefficients of the motor and cargo. This SDE system will be the fundamental object of study in this paper.

In Figure 2.3, we show realizations of the nondimensionalized version of (2.3) (specifically, the equations given in (3.2)) for two choices of parameters. It is easy to see that the motion of the complex is very regular - in particular, the time between 
steps of the motor has a very small variance. The overarching goal of this paper is to explain this phenomenon.
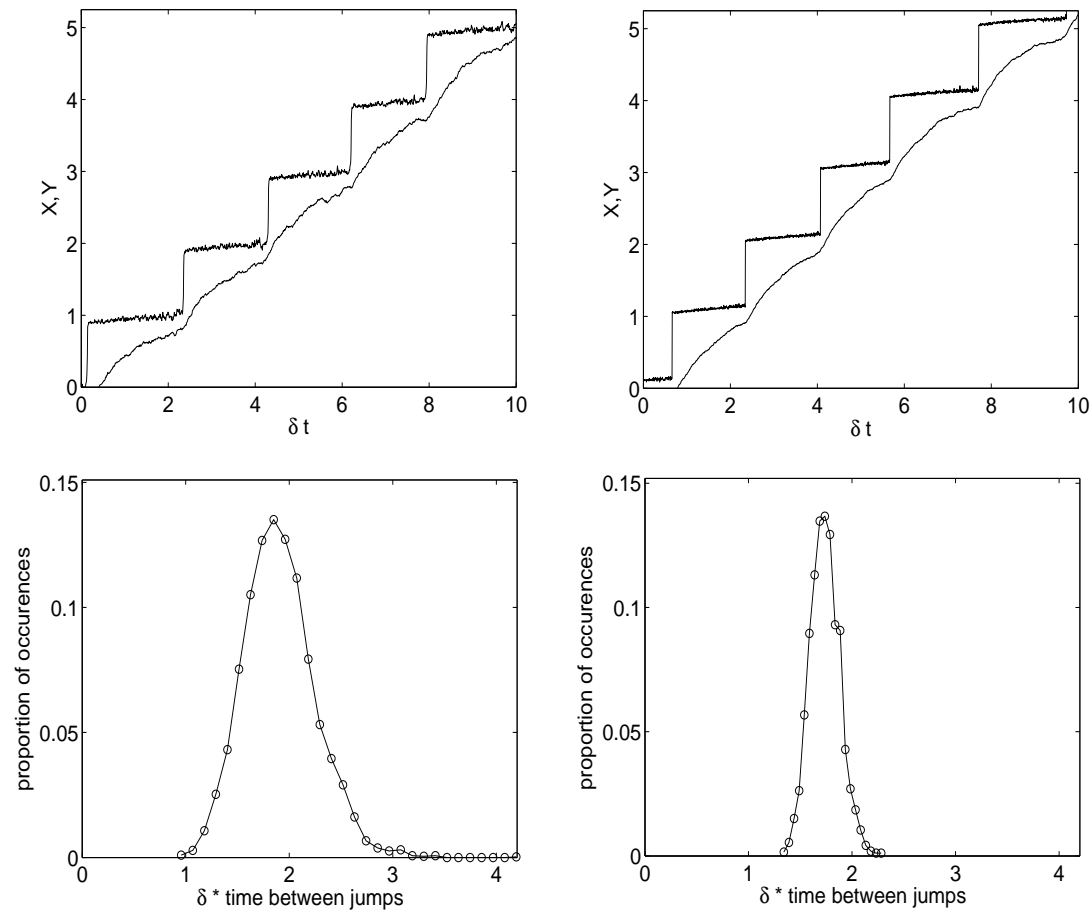

FIG. 2.3. In this figure we show realizations of (3.2) for two different choices of parameters, and histograms of their jumping times. In all simulations we have chosen $U(x)=(1-\cos (2 \pi x)) / 6.6-x$, $\alpha=3.3$, and $S(x-y)=(x-y)^{2} / 2$. The top left frame is a realization of (3.2) with $\epsilon=4 \times 10^{-3}$ and $\delta=8 \times 10^{-2}$, where we plot the position of a realization of $x$ and $y$ versus $\delta t$ (we show below that this is the natural timescale for the problem, see for example Theorem A.1). It can be observed that the motor stays pinned in a small region while the cargo relaxes towards it, and at some time before the cargo reaches the motor, the motor jumps to the next region. The bottom left frame shows a histogram of these residence times (more specifically, this is a histogram of the difference of the times where the trajectory crosses the integers). The right two frames are similar, except we have chosen $\epsilon=1 \times 10^{-3}$ and $\delta=4.5 \times 10^{-5}$. Notice that the second choice of parameters decreases the variance of the residence times without appreciably changing their mean (in fact, the mean goes from 1.91 in the left to 1.73 in the right, but the standard deviation is halved, from 0.345 to 0.153 ). The results of this paper explain all of these features. We point out that these simulations are done with the nondimensionalized equations (3.2) instead of the original SDE (2.3) to emphasize the limiting behavior which arises when $\delta, \epsilon \rightarrow 0$ in the particular distinguished limit considered in this paper.

\section{Asymptotic analysis and self-induced stochastic resonance}

In this section, we analyze (2.3) and show that in a particular distinguished scaling limit, the dynamics of the motor become completely regular. This section contains only a formal argument; we state and sketch the proof of a precise formulation of the limit in Appendix A.

The main idea behind the argument is that the motor moves in a potential which is the sum of the underlying potential given by $U$ and the coupling to the cargo given by $S$. As the cargo relaxes toward the motor, $S$ decreases and thus the potential well which the motor must escape to jump to the next tooth becomes less deep and hence 
less restrictive. We show that if the quantities are scaled correctly, there is a critical point where the timescale it takes for the motor to jump switches from being much longer than the relaxation timescale of the cargo to being much shorter. The motor jumps deterministically at this critical point.

In Section 3.1 we nondimensionalize (2.3) and discuss the parameters of interest. In Section 3.2 we propose a notational scheme and in Section 3.3 we describe the necessary large-deviation results and matching of timescales. In Section 3.4 and 3.5 we describe the dynamics of the system in two separate parameter regimes.

3.1. Rescaling and nondimensionalization. Recall that $S(|x-y|)$ is any convex function of $|x-y|$ with $S(0)=S^{\prime}(0)=0$. We assume for simplicity in this derivation that $x \geq y$, and we justify this assumption below. Plugging (2.1) into (2.3) gives

$$
\begin{aligned}
& d x=-\frac{D_{M}}{k_{B} T}\left(U^{\prime}(x)+S^{\prime}(x-y)\right) d t+\sqrt{2 D_{M}} d W_{t}^{x}, \\
& d y=\frac{D_{C}}{k_{B} T} S^{\prime}(x-y) d t+\sqrt{2 D_{C}} d W_{t}^{y} .
\end{aligned}
$$

We will rescale by

$$
x=A \hat{x}, \quad y=A \hat{y}, \quad t=B \hat{t},
$$

where $A$ is the period of the ratchet and $B$ is a timescale which we will set below. This gives

$$
\begin{aligned}
& d \hat{x}=\frac{D_{M} B}{k_{B} T A}\left(U^{\prime}(A \hat{x})+S^{\prime}(A(\hat{x}-\hat{y}))\right) d \hat{t}+\sqrt{\frac{2 D_{M} B}{A^{2}}} d \hat{W}_{t}^{x}, \\
& d \hat{y}=\frac{D_{C} B}{k_{B} T A} S^{\prime}(A(\hat{x}-\hat{y})) d \hat{t}+\sqrt{\frac{2 D_{C} B}{A^{2}}} d \hat{W}_{t}^{y} .
\end{aligned}
$$

We define $\kappa=S^{\prime \prime}(0)$ (this is the spring constant if the spring is linear) and set $B=k_{B} T / D_{M} \kappa$, and we introduce the dimensionless potentials:

$$
\hat{U}(\hat{x})=\frac{U(A \hat{x})}{U_{*}}, \quad \hat{S}(\hat{x})=\frac{S(A \hat{x})}{\kappa A^{2}} .
$$

This gives

$$
\begin{aligned}
& d \hat{x}=\left(\frac{U_{*}}{\kappa A^{2}} \hat{U}^{\prime}(\hat{x})+\hat{S}^{\prime}(\hat{x}-\hat{y})\right) d \hat{t}+\sqrt{\frac{2 k_{B} T}{\kappa A^{2}}} d \hat{W}_{t}^{x}, \\
& d \hat{y}=\frac{D_{C}}{D_{M}} \hat{S}^{\prime}(\hat{x}-\hat{y}) d \hat{t}+\sqrt{\frac{2 D_{C} k_{B} T}{D_{M} \kappa A^{2}}} d \hat{W}_{t}^{x} .
\end{aligned}
$$

Finally, we see that there are three nondimensional parameters remaining in the system, so we denote

$$
\alpha=\frac{U_{*}}{\kappa A^{2}}, \quad \delta=\frac{D_{C}}{D_{M}}, \quad \epsilon=\frac{k_{B} T}{\kappa A^{2}},
$$

and dropping all the hats for simplicity, we arrive at

$$
\begin{aligned}
& d x=-\left(\alpha U^{\prime}(x)+S^{\prime}(x-y)\right) d t+\sqrt{2 \epsilon} d W_{t}^{x}, \\
& d y=\delta S^{\prime}(x-y) d t+\sqrt{2 \epsilon \delta} d W_{t}^{y} .
\end{aligned}
$$


The parameter $\delta$ is the ratio of the diffusion coefficients of the cargo and motor. This paper considers the case where the cargo has a much smaller diffusion coefficient than the motor, giving $\delta \ll 1$. The parameter $\epsilon$ measures the energy accessible due to thermal effects relative to the amount of work it takes to stretch the spring by one period, and here we want to consider the case where this ratio is small, giving $\epsilon \ll 1$. The parameter $\alpha$ is the ratio of the energy released by the motor's moving one step forward in the ratchet relative to the amount of work it takes to stretch the spring by this distance. However, the particular meaning of $\alpha$ here is to quantify the relative strengths of the two potentials in which the motor moves (this can be seen from (3.2)), and thus $\alpha^{-1}$ corresponds to the nondimensionalized strength of the spring. For this paper, we will consider $\alpha$ to be a fixed $O(1)$ quantity subject to conditions described below. This will give an effective upper bound on the spring stiffness (we discuss this issue further in Section 4). Finally, note that $U$ has been rescaled in such a way that now $U(x+1)=U(x)-1$.

We mention that if the spring is linear, namely that $S(x-y)=\kappa(x-y)^{2} / 2$, then (3.2) becomes the simpler

$$
\begin{aligned}
& d x=-\left(\alpha U^{\prime}(x)+(x-y)\right) d t+\sqrt{2 \epsilon} d W_{t}^{x}, \\
& d y=\delta(x-y) d t+\sqrt{2 \epsilon \delta} d W_{t}^{y} .
\end{aligned}
$$

3.2. Notation. Consider (3.2) where we set $\delta=0$. This fixes $y$, and we see that $x(t)$ is simply diffusion in the potential given by

$$
V_{y}(x)=\alpha U(x)+S(x-y) .
$$

As derived above, $U(x)$ is a function which is periodic up to a shift, has local minima at the integers, and local maxima at $k+d$ for some $d \in(0,1), k \in \mathbb{Z}$, and $S$ is convex with $S(0)=S^{\prime}(0)=0$.

In particular, $V_{0}(x)$ has a local minimum at $x=0$. It follows from the implicit function theorem that there exists a neighborhood $N$ of 0 such that for all $y \in N$, the potential $V_{y}(x)$ has at least one local minimum. Moreover, the location of this local minimum can be chosen to depend smoothly on $y$. Let us define $x_{0}(y)$ to be the location of this local minimum, and define $\left(y_{\min }, y_{\max }\right)$ to be the maximal neighborhood for which this minimum exists. We will assume throughout that $V_{y}(x)$ has the property that $y_{\min }<-1$. Notice that for any choice of the motor potential $U$, we can guarantee this condition by choosing $\alpha$ sufficiently large (note further that this can be guaranteed by choosing the spring to be linear, and taking $\kappa$ sufficiently small while holding everything else fixed, as can be seen from (3.3)).

Also notice that the system (3.2) is invariant under the translation $(x, y) \mapsto(x+$ $k, y+k)$ for $k \in \mathbb{Z}$. We can thus define $x_{k}(y)=x_{0}(y-k)$ and it follows that for $y \in$ $\left(y_{\min }+k, y_{\max }+k\right)$, there exists a local minimum of $V_{y}(x)$ at $x_{k}(y)$. For topological reasons, there exists a unique local maximum between any two local minima. Thus, for any fixed $y \in\left(y_{\min }+k+1, y_{\max }+k\right)$, we define $u_{k}(y)$ to be the local maximum of $V_{y}(x)$ such that

$$
x_{k}(y)<u_{k}(y)<x_{k+1}(y)
$$

See Figures 3.1 and 3.2 for examples. Now, define

$$
\Delta V_{+}^{(k)}(y)=V_{y}\left(u_{k}(y)\right)-V_{y}\left(x_{k}(y)\right), \quad \Delta V_{-}^{(k)}(y)=V_{y}\left(u_{k-1}(y)\right)-V_{y}\left(x_{k}(y)\right)
$$




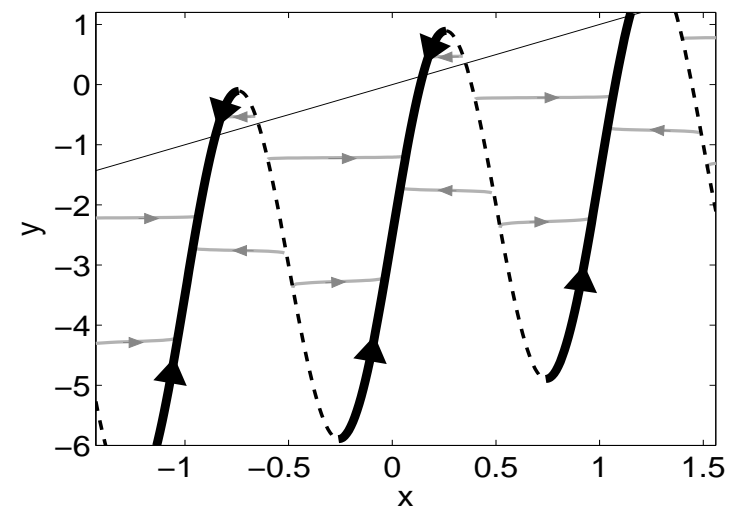

FIG. 3.1. The location of the local minima and maxima for $U(x)=(1-\cos (2 \pi x)) / 5-x, S(x)=$ $(x-y)^{2}$, and $\alpha=5$. We have graphed $x_{k}(y)$ with thick solid lines and $u_{k}(y)$ with dashed lines. The thin solid line is the line $y=x$, along which the system is translation invariant. We have also plotted several trajectories of the system (3.2) with $\epsilon=0$ (deterministic) and $\delta=10^{-1}$. Of course, the curves which are local minima for $V_{y}(x)$ become attracting slow manifolds for the deterministic system, and the local maxima likewise become repelling slow manifolds. All trajectories decay rapidly to an attracting slow manifold and then move along it, and of course we always move toward $y=x$ along any such slow manifold.
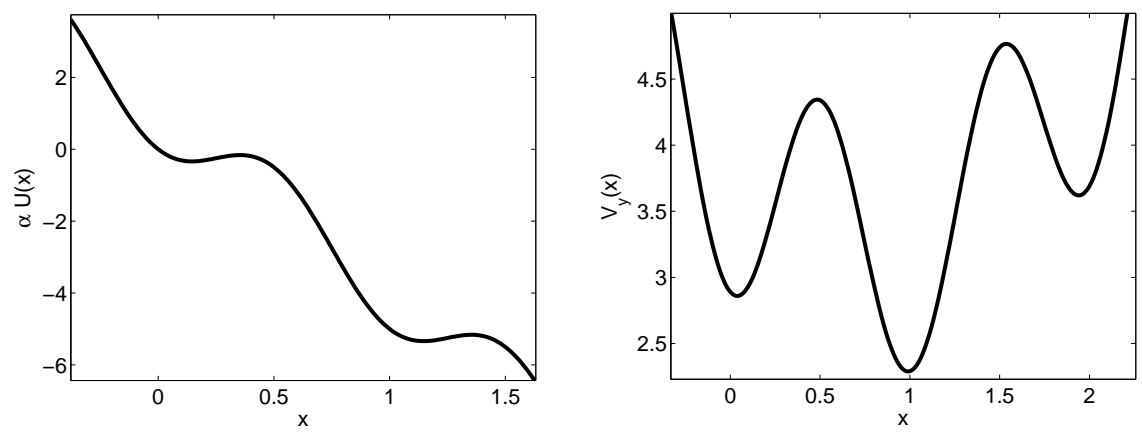

Fig. 3.2. Here we have chosen $U(x)=(1-\cos (2 \pi x)) / 5-x, \alpha=5$, and $S(x, y)=(x-y)^{2}$. In the left frame we have plotted $\alpha U(x)$, the ratchet potential, and in the right frame we have plotted the full potential $\alpha U(x)+S(x, y)$ with $y=-1.7$ (cf. the left frame and Figure 2.2). The three local minima which appear in the right panel are $x_{0}(-1.7), x_{1}(-1.7), x_{2}(-1.7)$, and the two local maxima are $u_{0}(-1.7), u_{1}(-1.7)$.

for all $y$ such that the right-hand sides exist. For any $k, \Delta V_{+}^{(k)}(y)$ is monotone decreasing in $y$ and $\Delta V_{-}^{(k)}(y)$ is monotone increasing in $y$, due to the convexity of the spring potential $S$. The interpretation of these two quantities is as follows: the local minimum $x_{k}(y)$ will have a local maximum to either side of it, and then $\Delta V_{+}^{(k)}(y)$ is the height of the barrier to the right of $x_{k}(y)$, and $\Delta V_{-}^{(k)}(y)$ is the height of the barrier to the left of $x_{k}(y)$.

3.3. Large-deviation asymptotics and matching. Throughout the remainder of this paper we use the notation $\left(X_{t}, Y_{t}\right)$ to denote a realization of $(3.2)$. 
Consider (3.2) with $y \in\left(y_{\min }+1,0\right)$ fixed (for the time being set $\left.\delta=0\right)$ and some initial condition $X_{0}=x$, with $x$ near $x_{0}(y)$. We will define $\tau_{R}^{(0)}(x, y)$ as the mean first exit time past $u_{0}(y)$, and $\tau_{L}^{(0)}(x, y)$ the mean first exit time past $u_{-1}(y)$. More specifically, choose some $h>0$ and define

$$
\begin{aligned}
& \tau_{R}^{(0)}(x, y)=\mathbb{E}_{x} \inf _{t>0}\left\{t:\left|X_{t}-x_{1}(y)\right|<h\right\}, \\
& \tau_{L}^{(0)}(x, y)=\mathbb{E}_{x} \inf _{t>0}\left\{t:\left|X_{t}-x_{-1}(y)\right|<h\right\} .
\end{aligned}
$$

Using the notation that $f(\epsilon) \asymp g(\epsilon)$ if and only if $\log (f(\epsilon)) / \log (g(\epsilon)) \rightarrow 1$ as $\epsilon \rightarrow 0$, it is a well-known result of large-deviation theory [13] that in the limit as $\epsilon \rightarrow 0$,

$$
\tau_{R}^{(0)}(x, y) \asymp \exp \left(\epsilon^{-1} \Delta V_{+}^{(0)}(y)\right), \quad \tau_{L}^{(0)}(x, y) \asymp \exp \left(\epsilon^{-1} \Delta V_{-}^{(0)}(y)\right) .
$$

Moreover, this limit is uniform for $x$ in any compact subset of $\left(u_{-1}(y), u_{1}(y)\right)$. We also point out that we can define activation energies and escape times for the other slow manifolds in a similar way by

$$
\begin{aligned}
\Delta V_{ \pm}^{(k)}(y) & =\Delta V_{ \pm}^{(0)}(y-k), \\
\tau_{R, L}^{(k)}(x, y) & =\tau_{R, L}^{(0)}(x-k, y-k),
\end{aligned}
$$

and by the translation invariance we also have

$$
\tau_{R}^{(k)}(x, y) \asymp \exp \left(\epsilon^{-1} \Delta V_{+}^{(k)}(y)\right), \quad \tau_{L}^{(k)}(x, y) \asymp \exp \left(\epsilon^{-1} \Delta V_{-}^{(k)}(y)\right) .
$$

Now, consider the effect of $\delta>0$, i.e. where we allow $y$ to move as well. We want to choose $\delta$ in such a way that the timescale on which $y$ relaxes is comparable to jumping events in $x$. This inspires us to consider the same limit as in $[11,6,7]$ :

$$
\epsilon \rightarrow 0, \quad \delta \rightarrow 0, \quad \epsilon \log \delta^{-1} \rightarrow \beta,
$$

where $\beta \in\left(\beta_{\min }, \beta_{\max }\right)$, with $\beta_{\min }$ and $\beta_{\max }$ to be specified below. This matching is equivalent to choosing $\delta \asymp e^{-\beta / \epsilon}$. We define $y_{\mathrm{f}}(\beta)$ and $y_{\mathrm{b}}(\beta)$ to be the unique solutions of

$$
\Delta V_{+}^{(0)}\left(y_{\mathfrak{f}}(\beta)\right)=\beta, \quad \Delta V_{-}^{(1)}\left(y_{\mathrm{b}}(\beta)\right)=\beta .
$$

Uniqueness follows from the monotonicity of $\Delta V_{ \pm}^{(k)}$. At least formally, we can see that in the limit $(3.8)$, the values $y_{\mathrm{f}}(\beta), y_{\mathrm{b}}(\beta)$ are the locations where we might expect the system to jump forward or backward between the slow manifolds $x_{0}(y)$ and $x_{1}(y)$, since these are the locations where the relaxation timescale of the cargo matches the jumping timescale of the motor (for example, $y_{\mathrm{f}}(\beta)$ is the value of $y$ for which the timescale to jump from $x_{0}(y)$ to $x_{1}(y)$ is the same as $\left.\delta^{-1}\right)$.

Finally, we assume throughout the rest of the paper that $\Delta V_{+}^{(0)}(0)<\Delta V_{-}^{(1)}(0)$, or, equivalently, that $V_{0}(0)>V_{0}(1)$. This will hold as long as we choose $\alpha$ sufficiently large in (3.4).

3.4. Non-stuttering dynamics. In this subsection, we will give an intuitive timescale-matching argument as to what the dynamics are with $\beta$ chosen sufficiently small. Define $\beta_{\mathrm{s}}$ to be the unique value of $\beta$ so that $y_{\mathrm{f}}(\beta)=y_{\mathrm{b}}(\beta)$ (see figure 3.3). In this section, we will assume that $\beta \in\left(\beta_{\min }, \beta_{\mathrm{s}}\right)$, where we define

$$
\beta_{\min }=\Delta V_{+}^{(0)}(0) \text {. }
$$




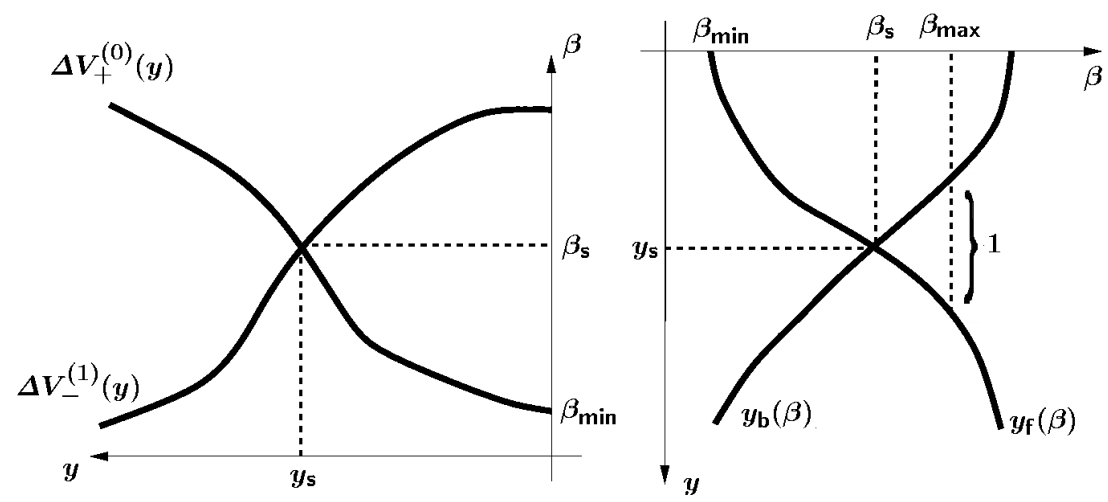

FIG. 3.3. The left frame shows the dependence of $\Delta V_{+}^{(0)}(y)$ and $\Delta V_{-}^{(1)}(y)$ on $y$, and the right shows the dependence of $y_{\mathrm{f}}(\beta)$ and $y_{\mathrm{b}}(b)$ on $\beta$. The monotonicity of these curves comes from the convexity of the spring potential $S$. The fact that $y_{\mathrm{s}}<0$, or equivalently, that $\beta_{\mathrm{s}}>\beta_{\min }$, is due to the monotonicity and to the assumption that $V_{0}(0)>V_{1}(0)$.
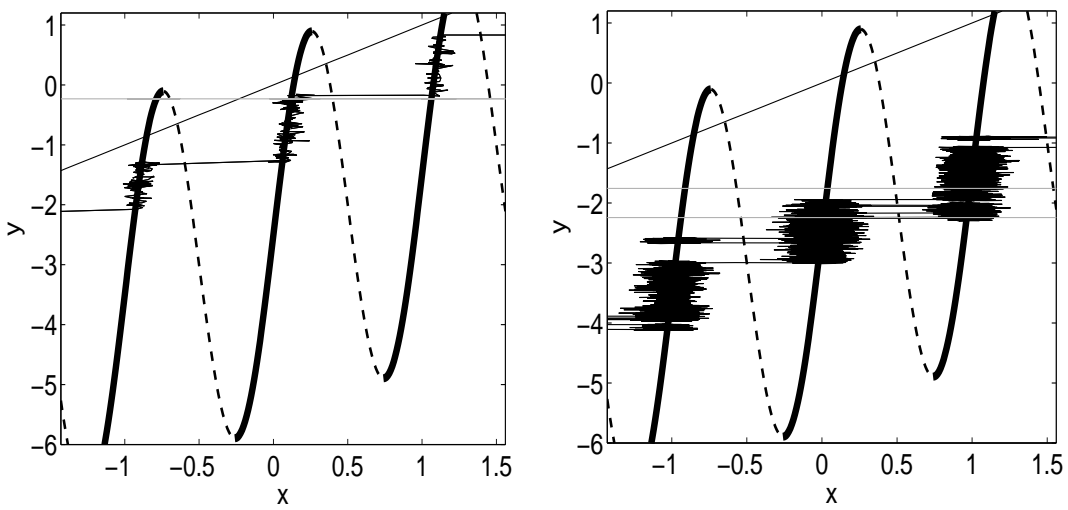

FIG. 3.4. Two simulations plotted in phase space, demonstrating both non-stuttering (the left panel) and stuttering (the right panel). Here we again use $U(x)=(1-\cos (2 \pi x)) / 5-x, \alpha=5$, and $S(x, y)=(x-y)^{2}$ (cf. the deterministic phase plane in Figure 3.1). Each panel shows a simulation of (3.2) with this choice of $U$ and $S$; the difference between the two panels is the choice of $\epsilon, \delta$. In the left panel, we choose $\epsilon=3 \times 10^{-2}, \delta=10^{-3}$; this gives an effective value of $\beta \approx 0.207$ (one can calculate that $\beta_{\mathrm{s}} \approx 0.881$ for this choice of $U$ and $S$ ) so that we expect not to stutter, and in fact we expect to jump off of $x_{0}(y)$ at $y_{\mathrm{f}} \approx-0.232$. (We have plotted $y=-0.232$ as a gray line in the left panel; notice that the trajectory jumps off of $x_{0}(y)$ very close to this line.) In the right panel, we choose $\epsilon=10^{-1}, \delta=4.54 \times 10^{-5}$, giving $\beta \approx 1.00$, so that we expect stuttering. One can further compute that for this $\beta$, we have $y_{\mathrm{f}} \approx-2.243, y_{\mathrm{b}} \approx-1.757$, so that the band of stuttering is approximately $(-2.243,-1.757)$. We plot the two lines $y=-2.243$ and $y=-1.757$ as gray lines in the right panel; notice that the trajectory seems to switch between $x_{0}(y)$ and $x_{1}(y)$ several times inside this band.

Referring again to Figure 3.3, note that the assumption made above that $V_{0}(0)>V_{1}(0)$ is sufficient to guarantee that $\beta_{\mathrm{s}}>\beta_{\mathrm{min}}$.

Moreover, it is equivalent to assume any of the following: that $\beta<\beta_{\mathbf{s}}$, that $\Delta V_{+}^{(0)}\left(y_{\mathfrak{f}}(\beta)\right)<\Delta V_{-}^{(1)}\left(y_{\mathfrak{f}}(\beta)\right)$, or that $y_{\mathfrak{f}}(\beta)>y_{\mathrm{b}}(\beta)$. 
For any $y \in\left(y_{\min }+1,0\right)$, it follows from (3.6) that for $\epsilon$ sufficiently small,

$$
\begin{cases}\tau_{R}^{(0)}(x, y) \gg \delta^{-1}, & y<y_{\mathrm{f}}(\beta), \\ \tau_{R}^{(0)}(x, y) \ll \delta^{-1}, & y>y_{\mathrm{f}}(\beta) .\end{cases}
$$

Now pick $y \in\left(y_{\mathrm{b}}(\beta), y_{\mathrm{f}}(\beta)\right)$, and consider any realization $\left(X_{t}, Y_{t}\right)$ of $(3.2)$ with initial condition $\left(X_{0}, Y_{0}\right)=\left(x_{0}(y), y\right)$. While $Y_{t} \in\left(y_{\mathrm{b}}(\beta), y_{\mathrm{f}}(\beta)\right)$, the time it takes for $X_{t}$ to jump to any neighborhood of $x_{1}\left(Y_{t}\right)$ or $x_{-1}\left(Y_{t}\right)$ is always much larger than $\delta^{-1}$, so a jump is very unlikely. Moreover, in the limit as $\epsilon \rightarrow 0, X_{t}$ spends an exponentially large fraction of time in any given neighborhood of $x_{0}(y)$.

In contrast, as soon as $Y_{t}>y_{\mathfrak{f}}(\beta)$, then $\tau_{R}^{(0)}\left(X_{t}, y\right) \ll \delta^{-1}$, so we expect the trajectory to move to a neighborhood of $x_{1}(y)$ quickly. Since $\Delta V_{-}^{(1)}(y)>\beta$, the probability of a backward jump, i.e. for $X_{t}$ to jump back to a neighborhood of $x_{0}\left(Y_{t}\right)$, is very small. Thus we expect the limiting process to move along the manifold $x_{0}(y)$ until $Y_{t}$ reaches $y_{\mathrm{f}}(\beta)$, at which time the process moves to a neighborhood of $x_{1}(y)$. Using the translation invariance of the system, the trajectory then moves along $x_{1}(y)$ until $Y_{t}$ reaches $y_{\mathbf{f}}(\beta)+1$, at which time it jumps to a neighborhood of $x_{2}(y)$, and this process repeats. See Figure 3.4 .

3.5. Stuttering dynamics. Let us now assume that $\beta>\beta_{\mathrm{s}}$ (again see Figure 3.3) and thus $y_{\mathrm{b}}(\beta)>y_{\mathrm{f}}(\beta)$. Thus for any fixed $y \in\left(y_{\mathfrak{f}}(\beta), y_{\mathrm{b}}(\beta)\right)$, we have both

$$
\tau_{R}^{(0)}(x, y) \ll \delta^{-1}, \quad \tau_{L}^{(1)}(x, y) \ll \delta^{-1} .
$$

We call this interval the "unstable region", simply because for $y$ in this region, we expect $X_{t}$ to make many switches between the two local minima at $x_{0}\left(Y_{t}\right)$ and $x_{1}\left(Y_{t}\right)$ in the time it takes $Y_{t}$ to appreciably change. Define

$$
\rho(y)=\frac{\tau_{R}^{(0)}(y)}{\tau_{R}^{(0)}(y)+\tau_{L}^{(1)}(y)},
$$

and define $y_{\mathrm{s}}$ by

$$
\Delta V_{+}^{(0)}\left(y_{\mathrm{s}}\right)=\Delta V_{-}^{(1)}\left(y_{\mathrm{s}}\right)
$$

The interpretation of $\rho$ is as follows: if we consider the first equation of (3.2) with fixed $y \in\left(y_{\mathrm{f}}(\beta), y_{\mathrm{b}}(\beta)\right)$, then $\rho(y)$ is the fraction of time we expect a realization to spend in a neighborhood of $x_{1}(y)$, and $(1-\rho(y))$ is the fraction of time it spends in a neighborhood of $x_{0}(y)$.

Using the formulas in (3.6), we see that, in the limit $\epsilon \rightarrow 0$,

$$
\rho(y) \rightarrow \begin{cases}0, & y \in\left(y_{\mathrm{f}}(\beta), y_{\mathrm{s}}\right), \\ 1, & y \in\left(y_{\mathrm{s}}, y_{\mathrm{b}}(\beta)\right) .\end{cases}
$$

Now consider the three intervals

$$
I_{1}(\beta)=\left(y_{\mathrm{b}}(\beta)-1, y_{\mathrm{f}}(\beta)\right), \quad I_{2}(\beta)=\left(y_{\mathrm{f}}(b), y_{\mathrm{s}}\right), \quad I_{3}(\beta)=\left(y_{\mathrm{s}}, y_{\mathrm{b}}(\beta)\right) .
$$

As before, in the limit $\epsilon \rightarrow 0$, we expect the process to escape any of these intervals in time which scales like $\delta^{-1}$, and to escape from the top with probability one. In fact, we expect the following: 
1. In $I_{1}(\beta)$, the trajectory stays near the slow manifold $x_{0}(y)$ at all times.

2. In $I_{2}(\beta)$, the trajectory spends an exponentially large fraction of time near $x_{0}(y)$. It escapes to a neighborhood of $x_{1}(y)$ in any small $y$ neighborhood, but returns quickly on the $\delta^{-1}$ timescale, since $V_{y}\left(x_{0}(y)\right)<V_{y}\left(x_{1}(y)\right)$.

3. In $I_{3}(\beta)$, similarly to above, the trajectory spends an exponentially large fraction of time near $x_{1}(y)$, but escapes to a neighborhood of $x_{0}(y)$ in any small $y$ neighborhood.

Then, as above, using the translation-invariance of the system and the formulas in (3.7), this repeats as follows: for $y \in\left(y_{\mathrm{s}}+k-1, y_{\mathrm{s}}+k\right)$, the system spends almost all of its time near the slow manifold $x_{k}(y)$, but for some band of values near $y_{\mathrm{s}}+k$ (which increases in size as $\beta$ increases), the trajectory makes many escapes to an adjacent slow manifold. See Figure 3.4. In particular, if we are only paying attention to where the system spends most of its time, stuttering is not qualitatively different from non-stuttering: it will spend most of its time near one manifold until the switch at $y_{\mathrm{s}}$, and even in the non-stuttering case the system still makes excursions away from the slow manifolds (just not excursions to neighborhoods of other slow manifolds). The stuttering only becomes apparent if we keep track of which manifold we are close to: then, the non-stuttering case only makes one transition between any two adjacent slow manifolds, and always does so in a forward manner, whereas the stuttering case makes many transitions between any adjacent slow manifolds. This distinction is made more precise in the limit theorems in Appendix A.

We want to define $\beta_{\max }$ so that for $\beta<\beta_{\max }$, these bands do not overlap. Thus we define $\beta_{\max }$ so that

$$
y_{\mathrm{b}}\left(\beta_{\max }\right)=y_{\mathrm{f}}\left(\beta_{\max }\right)+1 .
$$

One can see from Figure 3.3 that this ensures that

$$
\beta_{\min }<\beta_{\mathrm{s}}<\beta_{\max }
$$

We note in passing that the restriction $\beta<\beta_{\max }$ is artificial and made only for simplicity. One could choose $\beta>\beta_{\max }$, and then the motor would stutter amongst more than two adjacent domains (e.g. for $\beta$ just a bit above $\beta_{\max }$, the motor could sometimes stutter amongst three local minima of $V_{y}$ ). We do not consider this complication here, but of course the present results can be extended to that case as well.

\section{Conclusions}

We would like to conclude with a few comments on the properties of this model in the limit (3.8), and on what this might say for real motor protein models. In particular, we discuss the universality and the realizability of the analysis presented here.

We first discuss universality. Note that most details of the potential $U$ did not play any role in these calculations. Recall that we define $U$ so that it has local minima at integral multiples of the period $A$ and exactly one local maximum in the region $[0, A)$. Let $d \in[0, A)$ be the location of this local maximum. One can see that knowing the spring potential $S$ and the three parameters $d, U(d)-U(0)$ and $U_{*}$ is enough to fully specify the functions $\Delta V_{+}^{(k)}(y)$ and $\Delta V_{-}^{(k)}(y)$ completely, and in turn these specify the dynamics completely in the limit (3.8). Of course, what makes all of this work is that the matching which controls the dynamics only needs to consider the rough large-deviation estimates as in (3.7); since the prefactor corrections do not play a role here (as they only contribute an asymptotically small term in (3.8)), neither does 
the fine structure of the potential $U$. Thus the theory can be extended to much more complicated potentials than those considered here. In particular, it can be generalized to cases where the motor-cargo complex is described by a model with more than two degrees of freedom, to account at some level for the internal structure of the motor (see $[17,18,14,2])$. One particular generalization is non-smooth potentials; this can be achieved by taking a limit of smooth potentials where the three determining quantities are held constant. This is important because non-smooth potentials (e.g. piecewise-linear) are used in several applications [19, 9].

Second, we discuss some aspects of the realizability of the dynamics achieved here. The analysis above is applicable only in the distinguished limit (3.8), and in particular this implies that we are in the scaling limit $\delta \ll \epsilon \ll 1$. Here we repeat the definition of $\alpha, \delta, \epsilon$ from equation (3.1):

$$
\alpha=\frac{U_{*}}{\kappa A^{2}}, \quad \delta=\frac{D_{C}}{D_{M}}, \quad \epsilon=\frac{k_{B} T}{\kappa A^{2}} .
$$

Conversely, notice that as long as $\delta \ll \epsilon \ll 1$, we will be close to the limit (3.8) for some $\beta>0$. In particular, the meaning of $\epsilon \ll 1$ means that the work involved in one step of the motor is a significant multiple of $k_{B} T$.

It is observed [20] (see also [21]) or inferred [19] for multiple examples of motor proteins that the activation energy required to move the motor one step in the ratchet is about one order of magnitude greater than $k_{B} T$, but comparable to the energy released in the hydrolyzation of one ATP molecule. This means that $\alpha$ is $O(1)$, and $\epsilon$ is small enough so that $\epsilon \ll 1$, but, perhaps more importantly, it is not too small. Recall that for fixed $\beta, \delta$ is exponentially small in $\epsilon\left(\delta \asymp e^{-\beta / \epsilon}\right)$, and if $\epsilon$ were too small this would make the timescales on which any motion occurs inaccessible to the motor. Another view of this as it pertains to the choice of ratchet is that requiring $\alpha=O(1)$ and $\epsilon \ll 1$ means that we should have $U_{*} \gg k_{B} T$. But, again, if the motor is driven by ATP hydrolysis, then $U_{*} \approx 12 k_{B} T$ and we have $\epsilon$ one order of magnitude smaller than $\alpha$. Thus the choices of parameters for which we expect this limit to describe the dynamics of a molecular motor are of the same order of magnitude to those which have been measured.

The fact that $\epsilon$ is small for specific motor proteins suggests that regularity may be advantageous to their function. As was pointed out in [20], regularity in motor protein stepping could allow for a dense serial packing of identical motors along a filament. In the absence of any regularizing effect, such an array of motors would be likely to collide unless the packing were much more sparse. However, if they move regularly, they can move without collisions.

With a view towards experimental verification, we note that it is clear that in vitro experiments can be constructed in such a way as to test for the effect of changing $D_{C}$ and observing the dynamics of the motor-cargo complex. By taking $D_{C}$ small (i.e. high friction cargo) one can control the magnitude of $\delta$ and thus $\beta$. By controlling the magnitude of $D_{C}$ one can move the model from the stuttering regime to the non-stuttering regime, and vice versa. This adds another degree of falsification to these models, and it is quite interesting to see whether this observation is made in the future. It follows from the arguments in Appendix A that the velocity of (3.2) scales like $\delta^{-1}$, and converting this velocity back into the original variables gives

$$
v=\frac{A D_{C} \kappa}{k_{B} T} .
$$


Thus we see that taking $D_{C}$ small while holding everything else fixed slows down the velocity of the motor. However, it is reasonable to assume that $D_{M}$ can be made larger by modifying the solution in which the motor moves (e.g. by increasing the ATP concentration for a motor which uses ATP hydrolysis).

All of the above comments suggest that the limit considered in this paper may be realized by motor proteins in vivo. However, since it is not typically possible to modify the activation energy of a motor protein (which in this context implies that $\epsilon$ is inaccessible to experimenters), it will be useful to describe small (in $\epsilon$ ) perturbations to the dynamics described here. The authors have, in other contexts, considered perturbations to self-induced stochastic resonance in [4] and calculated corrections to the theory when the noise is taken to be small but positive. The authors are currently working on [8] in which these asymptotic effects are considered in real models of motor proteins.

Acknowledgments. The authors would like to thank Charles Peskin and Arjun Raj for several useful discussions. R.E.L.D. was supported as a Courant Instructor during this work. The work of E.V.-E. was partially supported by NSF via grant DMS02-39625, and by ONR via grant N00014-04-1-0565.

Appendix A. Theorem and proof. We want to state and prove precise statements corresponding to the intuitive arguments presented in Section 3. We first define the piecewise smooth trajectory to which the stochastic process limits. We then state and give a sketch of the proof of the limit theorem which corresponds to the dynamics described in Subsections 3.4 and 3.5. We point out that at the level of the first limit theorem, no distinction is made between the stuttering and non-stuttering cases; in fact, the stuttering is only in some sense evident when we coarse-grain the position of the motor into discrete bins. Finally, to make this distinction clearer, we state and prove the last theorem of this section.

Choose $y^{\star}<0$. For fixed $y^{\star}$, define $(\xi(t), \eta(t))$ as follows: let $\eta(t)$ solve

$$
\frac{d \eta}{d t}=S^{\prime}(\xi(t)-\eta(t)), \quad \xi(t)=x_{0}(\eta(t)),
$$

with initial condition $\eta(0)=y^{\star}-1$, for all $t \in\left[0, t^{\star}\right)$, where $t^{\star}$ is defined by $\eta\left(t^{\star}\right)=y^{\star}$. Equivalently, we can solve explicitly for $t^{\star}$ by

$$
t^{\star}=\int_{y^{\star}-1}^{y^{\star}} \frac{d \eta}{G(\eta)}, \quad G(y)=S^{\prime}\left(x_{0}(y)-y\right) .
$$

We extend this periodically: choose $k \in \mathbb{Z}$, and for $t \in\left[k t^{\star},(k+1) t^{\star}\right)$, let

$$
\xi(t)=\xi\left(t-k t^{\star}\right)+k, \quad \eta(t)=\eta\left(t-k t^{\star}\right)+k .
$$

This defines a piecewise smooth trajectory for all $t \in \mathbb{R}$. (The discontinuities are located at integral multiples of $t^{\star}$.)

We also point out that there were two restrictions needed in the arguments of Section 3: the first is that $y_{\min }<-1$, and the second is that $V_{0}(1)<V_{0}(0)$. Recall that both of these can be achieved by choosing $\alpha$ sufficiently large in (3.4).

Theorem A.1. Choose $\beta \in\left(\beta_{\min }, \beta_{\max }\right)$ where we define $\beta_{\min }, \beta_{\max }$ in (3.9) and (3.12). Assume that $V_{y}(x)$ is such that $y_{\min }<-1$ and $V_{0}(1)<V_{0}(0)$. Define $\delta=\delta(\epsilon)$ so that 
$\epsilon \log \delta^{-1}=\beta$, and choose $x, y \in \mathbb{R}$ with $y \leq x$. For each $\epsilon$, let $\left(X_{t}^{\epsilon}, Y_{t}^{\epsilon}\right)$ solve (3.2) with initial condition $X_{0}^{\epsilon}=x, Y_{0}^{\epsilon}=y$, and $\delta=\bar{\delta}(\epsilon)$. Define $\left(\hat{X}_{t}^{\epsilon}, \hat{Y}_{t}^{\epsilon}\right)=\left(X_{t / \delta}^{\epsilon}, Y_{t / \delta}^{\epsilon}\right)$ and $y^{\star}$ as

$$
y^{\star}= \begin{cases}y_{\mathrm{f}}(\beta), & \beta \in\left(\beta_{\min }, \beta_{\mathrm{s}}\right], \\ y_{\mathrm{s}}, & \beta \in\left(\beta_{\mathrm{s}}, \beta_{\max }\right) .\end{cases}
$$

and define $(\xi(t), \eta(t))$ as above. Then, for all $T>0, \eta>0$, there exists a phase shift $t_{0} \in \mathbb{R}$ such that

$$
\begin{aligned}
& \lim _{\epsilon \rightarrow 0} \operatorname{Prob}\left(\int_{0}^{T}\left|\hat{X}_{t}^{\epsilon}-\xi\left(t+t_{0}\right)\right|^{2} d t>\eta\right)=0, \\
& \lim _{\epsilon \rightarrow 0} \operatorname{Prob}\left(\sup _{t \in[0, T]}\left|\hat{Y}_{t}^{\epsilon}-\eta\left(t+t_{0}\right)\right|>\eta\right)=0 .
\end{aligned}
$$

Proof. [Sketch of proof.] With a few minor changes, the proof of this theorem is almost exactly the same as that given in [11]. In fact, the only difference between the setup in this paper and in [11] is that a stochastic term has been added to the slow variable, i.e. to the second equation in (3.2). A similar setup (in the context of multiscale Markov chains) with a noisy slow variable, which contains a proof with all details, is in [6].

For example, choose $\beta \in\left(\beta_{\min }, \beta_{\mathrm{s}}\right]$. Choose any initial condition with

$$
Y_{0} \in\left(y_{\mathfrak{f}}(\beta)-1, y_{\mathfrak{f}}(\beta)\right] \text { and } X_{0} \in\left(u_{0}\left(Y_{0}\right), u_{1}\left(Y_{0}\right)\right)
$$

(Recall that we have defined $u_{k}(y)$ in (3.5).) The argument in [11] starting at equation (11) and ending with the paragraph below (12) shows that as long as $Y_{t} \in\left(y_{\mathfrak{f}}(\beta)-1, y_{\mathfrak{f}}(\beta)\right)$, the probability of $X_{t}$ leaving the basin of attraction of $x_{0}(y)$, in time less than $O\left(\delta^{-1}\right)$, is exponentially small in $\epsilon$. Now, further note that if $X_{t}$ remains in this basin of attraction, the second equation in (3.2) has a positive drift, and thus as $\epsilon \rightarrow 0$, the probability that $Y_{t}$ exits the interval $\left(y_{\mathfrak{f}}(\beta)-1, y_{\mathfrak{f}}(\beta)\right)$ in time $O\left(\delta^{-1}\right)$, and does so through the top, goes to one. The convergence to the limiting trajectory as stated in the theorem follows in exactly the same manner as in [11].

Now assume $\beta \in\left(\beta_{\mathrm{s}}, \beta_{\min }\right)$. Recall the definitions of $I_{1}(\beta), I_{2}(\beta), I_{3}(\beta)$ as in $(3.11)$. Choose initial conditions $Y_{0} \in I_{1}(\beta)$ and $X_{0} \in\left(u_{0}\left(Y_{0}\right), u_{1}\left(Y_{0}\right)\right)$. The same argument as already given implies that $Y_{t}$ leaves $I_{1}(\beta)$ through the top, and that $X_{t}$ and $Y_{t}$ converge to the limiting trajectory in the manner prescribed in the theorem. Now assume that $Y_{0} \in I_{2}(\beta)$ and $X_{0} \in\left(u_{0}\left(Y_{0}\right), u_{1}\left(Y_{0}\right)\right)$. Now, in this case, since $\Delta V_{+}^{(0)}\left(Y_{t}\right)<\beta$, and thus $\tau_{R}^{(0)}\left(Y_{t}\right) \ll \delta^{-1}$, we expect $X_{t}$ to switch to a neighborhood of $x_{1}\left(Y_{t}\right)$ - in fact, by the same arguments as used in [11], for any $\Delta y>0$, and any neighborhood $\left(y_{0}-\Delta y, y_{0}+\Delta y\right)$ with $y_{0} \in I_{2}(\beta)$, it is easy to show that $X_{t}$ makes an excursion to a neighborhood of the slow manifold $x_{1}(y)$. However, the same large deviations estimates show that for $Y_{t} \in I_{2}(\beta), X_{t}$ spends an exponentially large fraction of time near the slow manifold $x_{0}(y)$. This is enough to show the convergence, as long as $y<y_{\mathrm{s}}-\Delta y$. The same argument shows convergence to the slow manifold $x_{1}(y)$ for $y \in\left(y_{\mathrm{s}}+\Delta y, y_{\mathrm{f}}(\beta)+1\right)$. It should be noted that for any $\epsilon>0$, we must take $\Delta y>0$ in the above argument, but as $\epsilon \rightarrow 0$, we can choose $\Delta y$ to go to zero as well.

As stated above, this limit theorem does not make any distinction between stuttering and non-stuttering. The main reason for this is the type of convergence one 
obtains in the fast direction. It can be shown (see $[6,11,12]$ for the statement and proof in similar problems) that for any of the allowable $\beta$, the convergence cannot be made uniform in the $x$-direction, and in fact it can be shown that there is a $O(1)$-neighborhood of the slow manifolds in which the trajectory is dense. From this perspective, the distinction between stuttering and non-stuttering is simply that of whether the size of this neighborhood includes two local minima of $V_{y}(x)$ instead of one, but this does not affect the limit since the system spends an exponentially large proportion of time near the minimum that is more stable. The real criterion distinguishing stuttering and non-stuttering is made apparent only if we think of the motor as sitting at a series of discrete sites - however, note that this is a typical perspective for motor protein models and Brownian ratchets in general, because these local minima are located at discrete states where the motor protein attaches to a filament.

We now make the distinction between the two cases more clear. Choose $\beta \in$ $\left(\beta_{\min }, \beta_{\max }\right)$ and let $\left(\hat{X}_{t}^{\epsilon}, \hat{Y}_{t}^{\epsilon}\right)$ be a realization of the stochastic process as in the previous theorem. Define the coarse-grained process $\tilde{X}_{t}$ by

$$
\tilde{X}_{t}^{\epsilon}=k \text { iff } \hat{X}_{t}^{\epsilon} \in\left(u_{k}\left(\hat{Y}_{t}^{\epsilon}\right), u_{k+1}\left(\hat{Y}_{t}^{\epsilon}\right)\right] .
$$

(In words, we are simply keeping track of which basin of attraction the motor sits in.) Define $\tilde{\xi}(t)=\left\lfloor\frac{t}{t^{\star}}\right\rfloor$. It follows from Theorem A.1 that $\tilde{X}_{t}^{\epsilon}$ converges to $\tilde{\xi}(t)$ (with an appropriate phase shift) in an $L^{2}$ sense. The distinction between stuttering or not is made by considering whether the convergence of the coarse-grained process is uniform:

Theorem A.2. Choose $\beta \in\left(\beta_{\min }, \beta_{\mathrm{s}}\right)$ and define the coarse-grained process $\tilde{X}_{t}^{\epsilon}$ and the coarse-grained limit $\tilde{\xi}(t)$ as above. Then there exists $t_{0} \in \mathbb{R}$ such that for any $T>0, h>0$, we have

$$
\lim _{\epsilon \rightarrow 0} \operatorname{Prob}\left(\sup _{t \in \mathcal{T}_{h}}\left|\tilde{X}_{t}^{\epsilon}-\tilde{\xi}\left(t+t_{0}\right)\right|>h\right)=0,
$$

where we define the set

$$
\mathcal{T}_{h}=[0, T] \backslash \bigcup_{k=0}^{\infty}\left(t^{\star}-h, t^{\star}+h\right) .
$$

In counterpoint, if we choose $\beta \in\left(\beta_{\mathbf{s}}, \beta_{\max }\right)$, then this convergence is not uniform. (In fact, more can be said: the relative measure of the set on which the coarse-grained process converges uniformly is less than one for $\beta \in\left(\beta_{\mathbf{s}}, \beta_{\max }\right)$ and goes to zero as $\beta \rightarrow \beta_{\max }$.)

Proof. [Sketch of proof.] The argument for this is really contained in the proof sketch above.

Choose $\beta \in\left(\beta_{\min }, \beta_{\mathrm{s}}\right]$. We know that we have the uniform convergence of $Y_{t}$ to $\eta(t)$ from the result above. By construction, $\xi(t)$ jumps in such a way that the inter-jump times go to $t^{\star}$ in the limit $\epsilon \rightarrow 0$. However, the argument above shows that for any $\Delta t>0$, the probability of $\tilde{X}_{t}$ switching basins of attraction outside of the set of times $\left[k t^{\star}-\Delta t, k t^{\star}+\Delta t\right]$ goes to zero exponentially fast as $\epsilon \rightarrow 0$. Combining this with the uniform convergence of $Y_{t}$ to $\eta(t)$ gives the result.

For $\beta \in\left(\beta_{\mathbf{s}}, \beta_{\max }\right)$, the proof of Theorem A.1 implies that anywhere in the "unstable region" $\left(y_{\mathrm{f}}(\beta), y_{\mathrm{b}}(\beta)\right), X_{t}$ is guaranteed to make many switches between $x_{0}\left(Y_{t}\right)$ and $x_{1}\left(Y_{t}\right)$, and this region is of size $O(1)$ even in the limit $\epsilon \rightarrow 0$, and thus uniform convergence is not possible. 


\section{REFERENCES}

[1] R. D. Astumian and I. Derenyi, Fluctuation driven transport and models of molecular motors and pumps, European Biophysics Journal with Biophysics Letters, 27(5), 474-489, 1998.

[2] P. J. Atzberger and C. S. Peskin, A Brownian dynamics model of kinesin in three dimensions incorporating the force-extension profile of the coiled-coil cargo tether, Bulletin of Mathematical Biology, 68(1), 131-160, 2006.

[3] N. Berglund and B. Gentz, Noise-induced Phenomena in Slow-Fast Dynamical Systems: A Sample-Paths Approach, Springer-Verlag, London, 2006.

[4] R. E. L. DeVille, C. B. Muratov and E. Vanden-Eijnden, Non-meanfield deterministic limits in chemical reaction kinetics far from equilibrium, Journal of Chemical Physics, 124(23), 231102, 2005

[5] R. E. L. DeVille, C. B. Muratov and E. Vanden-Eijnden, Two distinct mechanisms of coherence in randomly perturbed dynamical systems, Physical Review E (3), 72(3), 031105, 2005.

[6] R. E. L. DeVille and E. Vanden-Eijnden, Nontrivial scaling limits for multiscale Markov chains, J. Stat. Phys., 126(1), 75-94, 2007.

[7] R. E. L. DeVille and E. Vanden-Eijnden, Regularity and synchrony in motor proteins, Bulletin of Mathematical Biology, submitted, 2006.

[8] R. E. L. DeVille and E. Vanden-Eijnden, Stochastic analysis of a kinetic model for various molecular motors, in preparation.

[9] T. C. Elston and C. S. Peskin, The role of protein flexibility in molecular motor function: coupled diffusion in a titled periodic potential, SIAM J. Appl. Math., 60(3), 842-867, 2000.

[10] M. E. Fisher and A. B. Kolomeisky, The force exerted by a molecular motor, Proceedings of the National Academy of Sciences, 96(12), 6597-6602, 1999.

[11] M. I. Freidlin, On stable oscillations and equilibriums induced by small noise, J. Stat. Phys., 103(1-2), 283-300, 2001.

[12] M. I. Freidlin, On stochastic perturbations of dynamical systems with fast and slow components, Stochastics and Dynamics, 1(2), 261-281, 2001.

[13] M. I. Freidlin and A. D. Wentzell, Random Perturbations of Dynamical Systems, SpringerVerlag, New York, second edition, 1998.

[14] F. Jülicher, A. Adjari and J. Prost, Modeling molecular motors, Reviews of Modern Physics, 69(4), 1269-1281, 1997.

[15] M. Meister, S. R. Caplan and H. C. Berg, Dynamics of a tightly coupled mechanism for flagellar rotation-bacterial motility, chemiosmotic coupling, protonmotive force, Biophysical Journal, 55(5), 905-914, 1989.

[16] C. B. Muratov, E. Vanden-Eijnden and Weinan E, Self-induced stochastic resonance in excitable systems, Physica D, 210(3-4), 227-240, 2005.

[17] C. S. Peskin, G. M. Odell and G. F. Oster, Cellular motions and thermal fluctuations - the Brownian Ratchet, Biophysical Journal, 65(1), 316-324, 1993.

[18] C. S. Peskin and G. F. Oster, Coordinated hydrolysis explains the mechanical behavior of kinesin, Biophysical Journal, 68(4), S202-S210, 1995.

[19] A. Raj and C. S. Peskin, The influence of chromosome flexibility on chromosome transport during Anaphase A, Proceedings of the National Academy of Sciences of the United States of America, 103(14), 5349-5354, 2006.

[20] M. J. Schilstra and S. R. Martin, An elastically tethered viscous load imposes a regular gait on myosin- $V$, Journal of the Royal Society Interface, 3(6), 153-165, 2006.

[21] I. Schwaiger, C. Sattler, D. R. Hostetter and M. Rief, The myosin coiled-coil is a truly elastic protein structure, Nature Materials, 1(4), 232-235, 2002.

[22] S. M. Simon, C. S. Peskin and G. F. Oster, What drives the translocation of proteins? Proceedings of the National Academy of Sciences of the United States of America, 89(9), 3770-3774, 1992.

[23] H. Wang, T. Elston, A. Mogilner and G. Oster, Force generation in RNA polymerase, Biophysical Journal, 74(3), 1186-1202, 1997. 